Global Journal of Pure and Applied Mathematics.

ISSN 0973-1768 Volume 16, Number 6 (2020), pp. 783-788

(C) Research India Publications

https://dx.doi.org/10.37622/GJPAM/16.6.2020.783-788

\title{
Dealing with a Transportation Problem with Multi Choice Cost Coefficients and Fuzzy Supplies and Demands
}

\author{
Jadunath Nayak $^{* 1}$ and Srikumar Acharya ${ }^{2}$ \\ ${ }^{1}$ Baripada Degree College, Baripada, Odisha, India. E-mail-jadunath76@gmail.com \\ ${ }^{2}$ KIIT University, Bhubaneswar, Odisha, India. E-mail: e-mail-sacharyafma@kiit.ac.in
}

\begin{abstract}
In real life situations the parameters of Transportation problems are not certain. Either the parameters are fuzzy numbers or multiple choices are available. In this paper we consider a Transportation problem where the parameters like costs of transportation are multi choice in nature and other parameters like demands and supplies in the constrain are trapezoidal fuzzy numbers. We use binary variable approach to make a choice out of the available cost coefficients and ranking function is used to deal with trapezoidal fuzzy numbers. The problem was solved with existing methods and softwares like LINGO. A numerical example illustrate the purpose method.
\end{abstract}

Keywords: Transportation Problem, Multi choice Programming.

\section{INTRODUCTION}

A Transportation problem is a special type of linear programming problem in which we have to find out a suitable path between sources and destinations such that total cost of transportations is minimum. suppose there are $\mathrm{m}$ origin of sources and $\mathrm{n}$ destinations. let $a_{i}$ be the amount available at source $\mathrm{i}$ and $b_{j}$ be need of destination $\mathrm{j}$. Let $C_{i j}$ be the cost of transportation of a unit from $i^{\text {th }}$ source to $j^{\text {th }}$ destination. The problem is to find out the value $x_{i j}$ which is the amount to be transported from source $i$ to destination $\mathrm{j}$ such that the total cost of transportation is minimum. Many algorithm are available to solve this problem when the value $a_{i}, b_{j}, C_{i j}$ are crisp in nature.

*Corresponding Author 
In real life situations these factors are uncertain. After development of Fuzzy[6] concept a powerful tool was found to deal with uncertainties and a lot of developments was possible and still going on. Another way to deal with uncertainties is the concept of multi choices ( i.e. to make a suitable choice out of all available choices). Healy[3] was first to develop a procedure for linear programming problem with zero- one variable which is called multi choice programming. This gave another dimension to deal with uncertainties involved in Transportation problem. Biswal and Acarya[2] used binary variable to transform a multi-choice linear programming problem to a non- linear mixed integer programme. Kanan et.al. use this technique on Goal programming. Similarly Nayak et.al[4] suggest some techniques to solve Transportation problem with multi choice parameters.

Our considered problem is a mixture of multi choice factors and fuzzy parameters(in the form of trapezoidal fuzzy numbers), the multi choice parameters are deal using binary variables and trapezoidal fuzzy numbers are converted to crisp numbers using ranking function defined by Hadi BasirZadeh[1]

\section{MATHEMATICAL MODEL}

Consider a Transportation problem with $m$ numbers of origin (sources) and $n$ numbers of destination. Let $\tilde{a}_{i}$ be the fuzzy availability at $i^{\text {th }}$ destination and $\tilde{b}_{j}$ be the fuzzy requirement at $j^{\text {th }}$ destination, where $\tilde{a_{i}}$ and $\tilde{b_{j}}$ are trapezoidal fuzzy numbers represented by $\tilde{a_{i}}=\left(a_{i 1}, a_{i 2}, a_{i 3}, a_{i 4}\right)$ and $\tilde{b_{j}}=\left(b_{1 j}, b_{2 j}, b_{3 j}, b_{4 j}\right)$. let $\left\{C_{i j}^{(1)}, C_{i j}^{(2)} \ldots, C_{i j}^{\left(k_{i j}\right)}\right\}$ be multi choice cost co efficient for transporting a unit from $i^{\text {th }}$ source to $j^{\text {th }}$ destination. Now the mathematical model is represented as:

$$
\min : Z=\sum_{i=1}^{n} \sum_{j=1}^{n}\left\{C_{i j}^{(1)}, C_{i j}^{(2)} \ldots, C_{i j}^{\left(k_{i j}\right)}\right\} x_{i j}
$$

Subject to

$$
\begin{aligned}
\sum_{i=1}^{m} x_{i j} & \leq \tilde{a_{i}} \\
\sum_{j=1}^{n} X_{i j} & \geq \tilde{b_{j}} \\
x_{i j} & \geq 0
\end{aligned}
$$

This problem can not solved in present form. We have to find out an equivalent model to solve this problem using following techniques.

\section{EQUIVALENT MODEL}

Every natural number can be express as sum of $2^{k}$ number of terms and each term is a power of 2 , where $k \in \mathbb{N} \cup\{0\}$, hence using this principle we can use suitable numbers 
of binary variables to convert the objective functions. The methods of considering binary variables are discuss by Nayak et.al[4] and kanan et.al[5]. The fuzzy demands and supplies are converted to crisp value using the method suggested bu Hadi[1].which stated as

If $(a, b, c, d)$ is a trapezoidal fuzzy number then

$$
\begin{array}{r}
\left.M_{0}^{T r a}\{B\}=\frac{1}{2} \int_{0}^{1}\left\{B(r)+B^{(} r\right)\right\} d r \\
=\frac{1}{4}\{a+b+c+d\}
\end{array}
$$

Then the problem can be stated as.

$$
\min : Z=\sum_{i=1}^{n} \sum_{j=1}^{n} P\left(\theta_{i j}^{(1)}, \theta_{i j}^{(2)}\right) x_{i j}
$$

subject to

$$
\begin{aligned}
& \sum_{i=1}^{n} x_{i j} \leq M_{O}^{T r a}\left(a_{i}\right), \quad i=1,2, \cdots, n \\
& \sum_{j=1}^{n} x_{i j} \leq M_{O}^{T r a}\left(b_{j}\right), \quad j=1,2, \cdots, m \\
& \sum_{i=1}^{n} \sum_{j=1}^{n} X_{i j}=\min \left\{\sum M_{0}^{\text {Tra }}\left(a_{i}\right), \sum M_{O}^{T r a}\left(b_{j}\right)\right\} \\
& x_{i j} \geq 0 \geq 0 \\
& \theta_{i j}^{(1)}, \theta i j^{(2)} \in\{0,1\}
\end{aligned}
$$

The above problem can be solve using existing methods and software like LINGO.

\section{NUMERICAL EXAMPLE}

In this section we used a numerical example to illustrate the proposed method.

\subsection{Example}

Consider a transportation problem where the supply and demands are trapezoidal fuzzy number and there are multi choice for each cost of transportation. 


\begin{tabular}{|c|c|c|c|c|c|}
\hline & $S_{1}$ & $S_{2}$ & $S_{3}$ & $S_{4}$ & Supply \\
\hline$D_{1}$ & $\{3,4,7\}$ & $\{4,5,6\}$ & $\{9,10\}$ & $\{7,8\}$ & $(7,8,9,10)$ \\
\hline$D_{2}$ & $\{3,4,6\}$ & $\{2,3\}$ & $\{1,4,2\}$ & $\{6,4,5\}$ & $(12,14,16,17)$ \\
\hline$D_{3}$ & $\{1,4,3\}$ & $\{2,4\}$ & $\{3,5\}$ & $\{10,11\}$ & $(10,13,14,15)$ \\
\hline Demand & $(5,6,7,8)$ & $(10,11,12,13)$ & $(8,10,11,12)$ & $(6,7,9,10)$ & \\
\hline
\end{tabular}

\subsection{Solution}

We first convert all trapezoidal fuzzy number to crisp number by using 3.5.

$$
\begin{aligned}
& M_{O}\left(a_{1}\right)=\frac{7+8+9+10}{4}=8.5 \\
& M_{O}\left(a_{2}\right)=\frac{12+14+16+17}{4}=14.75 \\
& M_{O}\left(a_{3}\right)=\frac{10+13+14+15}{4}=13 \\
& M_{O}\left(b_{1}\right)=\frac{5+6+7+8}{4}=6.5 \\
& M_{O}\left(b_{2}\right)=\frac{10+11+12+13}{4}=11.5 \\
& M_{O}\left(b_{3}\right)=\frac{8+10+11+12}{4}=10.25 \\
& M_{O}\left(b_{4}\right)=\frac{6+7+9+10}{4}=8
\end{aligned}
$$

The cost coefficients can be converted using binary variables.

$$
\theta_{11}^{1}, \theta_{11}^{2}, \theta_{12}^{1}, \theta_{12}^{2}, \theta_{13}^{1}, \theta_{13}^{2}, \theta_{14}^{1}, \theta_{14}^{2}, \theta_{21}^{1}, \theta_{21}^{2}, \theta_{22}, \theta_{23}^{1}, \theta_{23}^{2}, \theta_{24}^{1}, \theta_{24}^{2}, \theta_{31}^{1}, \theta_{31}^{2}, \theta_{32}, \theta_{33}, \theta_{34}
$$

we can convert the cost co-efficient as

$$
\begin{aligned}
& C_{11}=3 \theta_{11}^{1} \theta_{11}^{2}+4 \theta_{11}^{1}\left(1-\theta_{11}^{2}\right)+7\left(1-\theta_{11}^{1}\right) \theta_{11}^{2} \\
& C_{12}=4 \theta_{12}^{1} \theta 12^{2}+5 \theta_{12}^{1}\left(1-\theta_{12}^{2}\right)+6\left(1-\theta_{12}^{1}\right) \theta_{12}^{2} \\
& C_{13}=9 \theta_{13}+10\left(1-\theta_{13}\right) \\
& C_{14}=7 \theta_{14}+8\left(1-\theta_{14}\right) \\
& C_{21}=3 \theta_{21}^{1} \theta 21^{2}+4 \theta_{21}^{1}\left(1-\theta_{21}^{2}\right)+6\left(1-\theta_{21}^{1}\right) \theta_{21}^{2} \\
& C_{22}=2 \theta_{22}+3\left(1-\theta_{22}\right) \\
& C_{23}=1 \theta_{23}^{1} \theta_{23}^{2}+4 \theta_{23}^{1}\left(1-\theta_{23}^{2}\right)+2\left(1-\theta_{23}^{1}\right) \theta_{23}^{2} \\
& C_{24}=6 \theta_{24}^{1} \theta_{24}^{2}+4 \theta_{24}^{1}\left(1-\theta_{24}^{2}\right)+5\left(1-\theta_{24}^{1}\right) \theta_{24}^{2} \\
& C_{31}=1 \theta_{31}^{1} \theta 31^{2}+4 \theta_{31}^{1}\left(1-\theta_{31}^{2}\right)+3\left(1-\theta_{31}^{1}\right) \theta_{31}^{2} \\
& C_{32}=2 \theta_{32}+4\left(1-\theta_{32}\right) \\
& C_{33}=3 \theta_{33}+5\left(1-\theta_{33}\right) \\
& C_{34}=10 \theta_{34}+11\left(1-\theta_{34}\right)
\end{aligned}
$$


Using above replacement the problem reduce to.

$$
\begin{aligned}
\min : Z & =\left(3 \theta_{11}^{1} \theta 11^{2}+4 \theta_{11}^{1}\left(1-\theta_{11}^{2}\right)+7\left(1-\theta_{11}^{1}\right) \theta_{11}^{2}\right) X_{11}+\left(4 \theta_{12}^{1} \theta 12^{2}+5 \theta_{12}^{1}\left(1-\theta_{12}^{2}\right)\right. \\
& \left.+6\left(1-\theta_{12}^{1}\right) \theta_{12}^{2}\right) X_{12}+\left(9 \theta_{13}+10\left(1-\theta_{13}\right)\right) X_{13}+\left(7 \theta_{14}+8\left(1-\theta_{14}\right)\right) X_{14} \\
& +\left(3 \theta_{21}^{1} \theta 21^{2}+4 \theta_{21}^{1}\left(1-\theta_{21}^{2}\right)+6\left(1-\theta_{21}^{1}\right) \theta_{21}^{2}\right) X_{21}+\left(2 \theta_{22}+3\left(1-\theta_{22}\right)\right) X_{22} \\
& +\left(1 \theta_{23}^{1} \theta_{23}^{2}+4 \theta_{23}^{1}\left(1-\theta_{23}^{2}\right)+2\left(1-\theta_{23}^{1}\right) \theta_{23}^{2}\right) X_{23}+\left(6 \theta_{24}^{1} \theta_{24}^{2}\right. \\
& \left.+4 \theta_{24}^{1}\left(1-\theta_{24}^{2}\right)+5\left(1-\theta_{24}^{1}\right) \theta_{24}^{2}\right) X_{24}+\left(1 \theta_{31}^{1} \theta 31^{2}+4 \theta_{31}^{1}\left(1-\theta_{31}^{2}\right)+3\left(1-\theta_{31}^{1}\right) \theta_{31}^{2}\right) X_{31} \\
& +\left(2 \theta_{32}+4\left(1-\theta_{32}\right)\right) X_{32}+\left(3 \theta_{33}+5\left(1-\theta_{33}\right)\right) X_{33}+\left(10 \theta_{34}+11\left(1-\theta_{34}\right)\right) X_{34}
\end{aligned}
$$

Subject to

$$
\begin{gathered}
X_{11}+X_{12}+X_{13}+X_{14} \leq 8.5 \\
X_{21}+X_{22}+X_{23}+X_{24} \leq 14.75 \\
X_{31}+X_{32}+X_{33}+X_{34} \leq 13 \\
X_{11}+X_{21}+X_{31} \geq 6.5 \\
X_{12}+X_{22}+X_{32} \geq 11.5 \\
X_{13}+X_{23}+X_{33} \geq 10.25 \\
X_{14}+X_{24}+X_{34} \geq 8 \\
\\
\theta_{11}^{1}, \theta_{11}^{2}, \theta_{12}^{1}, \theta_{12}^{2}, \theta_{13}, \theta_{14}, \theta_{21}^{1}, \theta_{21}^{2}, \theta_{22}, \theta_{23}^{1}, \theta_{23}^{2}, \theta_{24}^{1}, \theta_{24}^{2}, \theta_{31}^{1}, \theta_{31}^{2} \theta_{32}, \theta_{33}, \theta_{34} \in\{0,1\} \\
\text { the above problem can be solved using LINGO and the result is } \\
\theta_{11}^{1}=1, \theta_{11}^{2}=1, \theta_{12}^{1}=1, \theta_{12}^{2}=1, \theta_{13}=1, \theta_{14}=1, \theta_{21}^{1}=1, \theta_{21}^{2}=1, \theta_{22}=1, \theta_{23}^{1}= \\
1, \theta_{23}^{2}=1, \theta_{24}^{1}=0, \theta_{24}^{2}=0, \theta_{31}^{1}=1, \theta_{31}^{2}=1, \theta_{32}=1, \theta_{33}=1, \theta_{34}=0, C_{11}=3, C_{12}= \\
4, C_{13}=9, C_{14}=7, C_{21}=3, C_{22}=2, C_{23}=1, C_{24}=6, C_{31}=1, C_{32}=2, C_{33}=3, C_{34}= \\
11, X_{11}=0, X_{12}=8.5, X_{13}=0, X_{14}=0, X_{21}=0, X_{22}=0, X_{23}=6.5, X_{24}=8, X_{31}= \\
6.5, X_{32}=3, X_{33}=3.5, X_{34}=0 \text { and the optimal cost of transportation is } 63.75 .
\end{gathered}
$$

\section{RESULT DISCUSSION AND CONCLUSION}

We used suggested method to solve a transportation problem with fuzzy demand, fuzzy supply and multi-choice cost co-efficient. Further research is reflect the more effective methods for converting multi-choice value to an equivalent value. Linearization of binary variable may be taken as further research. Similarly interpolating polynomial can be use to handel multi choice parameters.

\section{REFERENCES}

[1] Hadi Basirzadeh. An approach for solving fuzzy transportation problem. Applied Mathematical Sciences, 5(32):1549-1566, 2011.

[2] MP Biswal and S Acharya. Transformation of a multi-choice linear programming problem. Applied Mathematics and Computation, 210(1):182-188, 2009. 
[3] WC Healy Jr. Multiple choice programming (a procedure for linear programming with zero-one variables). Operations Research, 12(1):122-138, 1964.

[4] Jadunath Nayak, Sudarsan Nanda, and Srikumar Acharya. Generalized binary variable approach to solve multi-choice transportation problem. International Journal of Engineering, Science and Mathematics, 6(5):158-165, 2017.

[5] Kanan K Patro, MM Acharya, MP Biswal, and Srikumar Acharya. Computation of a multi-choice goal programming problem. Applied Mathematics and Computation, 271:489-501, 2015.

[6] Lotfi A Zadeh. Fuzzy sets. Information and control, 8(3):338-353, 1965. 